\title{
Cerebrospinal Fluid Otorrhea Caused by Arachnoid Granulation
}

\author{
Sang Woo Kim and Jeong Hwan Choi \\ Department of Otolaryngology Head and Neck Surgery, Sanggye Paik Hospital, College of Medicine, Inje University, Seoul, Korea
}

\author{
Received May 28, 2012 \\ Revised September 18, 2012 \\ Accepted September 22, 2012
}

\author{
Address for correspondence \\ Jeong Hwan Choi, MD \\ Department of Otolaryngology \\ Head and Neck Surgery, \\ Sanggye Paik Hospital, \\ College of Medicine, Inje University, \\ 1342 Dongil-ro, Nowon-gu, \\ Seoul 139-707, Korea \\ Tel +82-2-950-1104 \\ Fax +82-2-935-6220 \\ E-mail choijh92@paik.ac.kr
}

Cerebrospinal fluid (CSF) leakage otorrhea may be congenital or can be caused by trauma, surgery, cholesteatoma, and tumors. Spontaneous CSF leakage through the middle ear without a secondary cause is a relatively rare disease. The pathophysiology of CSF otorrhea has not been clear yet. However, there are two theories of the pathophysiology of spontaneous CSF otorrhea have been studied in the medical field: one based on the congenital defect; the other about the arachnoid granulation which causes bone erosion. Herein, we examine and report a case of CSF otorrhea caused by arachnoid granulation. Literatures pertaining to the disorder will be reviewed and characteristics of the disorder also will be discussed.

Korean J Audiol 2012;16:152-155

KEY WORDS: Cerebrospinal fluid otorrhea · Arachnoid · Granulation tissue.

\section{Introduction}

Cerebrospinal fluid (CSF) otorrhea, which is occurred by secondary causes, such as a congenital development disorder of inner ear, surgery, trauma or cholesteatoma, is generated from a fistula that is formed when the barriers between the mastoid part of the temporal bone and the bone matrix of skull are breakdown. ${ }^{1)}$ The fistula may causes meningitis by connecting the ear cavity and the intracranial space. Spontaneous CSF with middle ear effusion and otorrhea were reported by Canfield $^{2)}$ in 1913 for the first time. The patients are mostly over age 40, and it is more likely that they don't show specific ear symptoms. ${ }^{3)}$ Currently, the pathophysiology of CSF otorrhea has not been clear yet. However, there are two theories of the pathophysiology of spontaneous CSF otorrhea have been suggested. One is based on the congenital defect and the other is about the arachnoid granulation which causes bone erosion. ${ }^{4}$

We report a case of CSF otorrhea caused by arachnoid granulation with the reviewed literatures.

\section{Case Report}

A 45 year-old female patient was referred to our depart- ment with right ear fullness which was precipitated by changes in position. Her symptom was initiated about 3 months ago. She has been well controlled hypertension. In physical examination, air fluid level was found in her right tympanic membrane, and the level increased when she hung her head down. Pure tone audiometry examination showed the right conductive hearing loss. Impedance test showed that the right was $\mathrm{C}$ type and the left was A type. In order to distinguish CSF otorrhea, her middle ear fluid was aspirated, and its glucose level noted $88 \mathrm{mg} / \mathrm{dL}$. Computed tomogram (CT) of temporal bone demonstrated a bony defect adjacent to the soft tissue lesion of posterior wall of the temporal bone with an effusion showing high attenuation in the right mastoid cavity (Fig. 1). Thus, we presumed that CSF otorrhea might be caused by arachnoid granulation. She underwent mastoidectomy under general anesthesia. During a procedure for canal wall-up mastoidectomy, in accordance with the findings of the CT scan, the tissue which was suspected to be arachnoid granulation was observed with the bony defect of the posterior wall. The surrounding bone was removed by a diamond drill. The granulation tissue was placed at the defect of meninx (Fig. 2). The tissue was obtained for a biopsy. The remained granulation tissue was shrunk by bipolar cauterization. The bony defect was reconstructed by the auricular cartilage and perichondrium complex graft. 
Subsequently, fibrin glue was placed over the graft. The biopsy demonstrated a fibrillary tissue (Fig. 3A). Moreover, glial fibrillary acidic protein stain indicated a positive reaction, and we confirmed arachnoid granulation which contained a portion of parenchyma of the cerebrum (Fig. 3B). The patient has not had any symptoms of the disorder. In addition, 1 year and 3 months after the surgery, she's been continuing follow up in the outpatient clinics without any complications or recurrence.

\section{Discussion}

CSF otorrhea is most frequently occurred by secondary causes including surgery, tumors, and infection but it can be spontaneously occurred. ${ }^{5,6)}$ Children are subject to spontaneous CSF otorrhea, and it can be detected with congenital disorders, such as Mondini anomaly, patent cochlear aqueduct, patent Hyrtl fissure, patent petromastoid canal, and patulous facial canal. ${ }^{5-7)}$ Spontaneous CSF otorrhea can be also detected in adults, and it occurs when the bone matrix, which is between the middle ear and the intracranial space, is abnormal. Arachnoid granulation is also a cause of craniodural defect. ${ }^{4,5,8)}$
When CSF leaks through the ear or the nose, critical complications, such as bacterial meningitis can be developed. Therefore early diagnosis and appropriate surgical managements are critical. Arachnoid granulation is a herniation of the arachnoid tissue from the dural defect into the dural sinus. ${ }^{4,9)}$ At gestational age of 36 weeks, arachnoid granulation formed

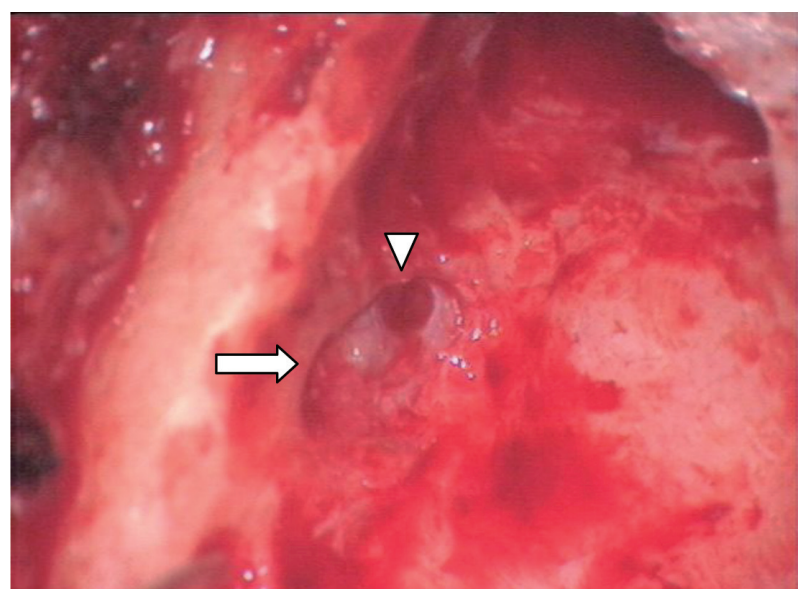

Fig. 2. Craniodural defect at the operation field. The bony defect (arrow) was enlarged by drilling. After arachnoid granulation was removed, the defect at the dura (arrow-head) was seen.
Fig. 1. Bony defect on posterior wall of right temporal bone (white arrow) and mastoid effusion were seen at the temporal bone CT axial view (A) and coronal view (B).

Fig. 3. Fibrillary tissues indicated a brain component (A, H\&E stain, $\times 40)$. Glial fibrillary acidic protein (GFAP) stain showed a positive reaction. Thus, it meant that the origin of the tissue was the central nervous system (B, GFAP stain, $\times 400$ ).
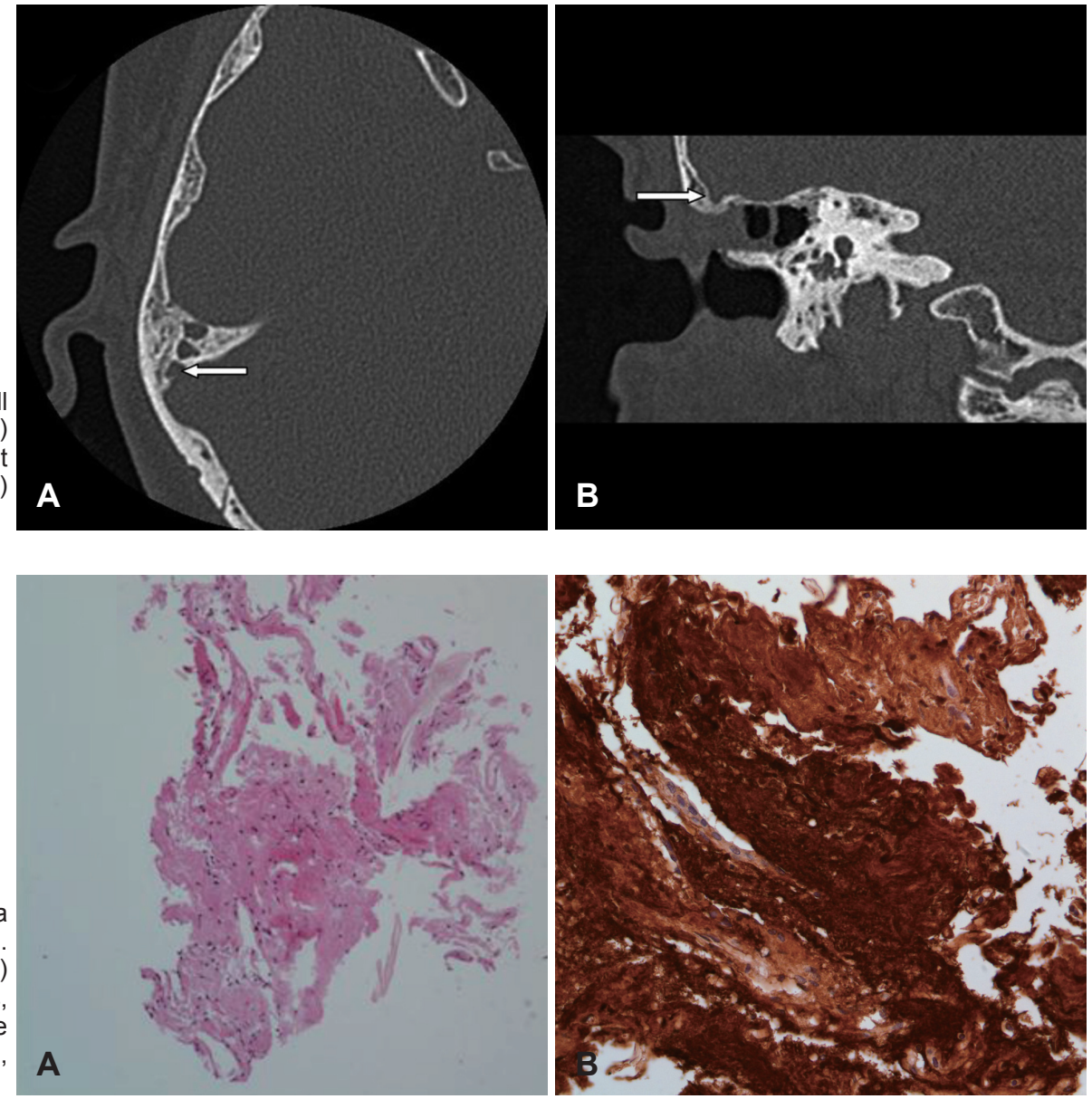
in the vili of arachnoid membrane becomes enlarged and lobulated as it gets old and increased the CSF pressure. Since CSF pressure is higher than venous pressure, CSF flows from the subarachnoid space into the intracranial venous system through gaps between the endothelial cells covering the arachnoid granulation and pinocytosis. ${ }^{10)}$ Most arachnoid granulation is distributed in the areas of the venous sinus or the bone matrix vein. In a case of the temporal bone, arachnoid granulation is formed toward the sigmoid sinus. When capsulation is occurred by ossification of mesenchyme in an early stage, it doesn't expand toward the sinus without ossification, it becomes covered only with thin fibrous coat. When the CSF pressure in the center of arachnoid granulation gets higher and penetrates a part of the dura, it meets the bone matrix of skull. Further, if it is exposed for long, it may cause bone erosion., ${ }^{5,11}$ As a result of that, if there is the dural injury and the temporal bone defect, the CSF otorrhea will occurs eventually.

In radiologic study reports, the frequency of arachnoid granulation ranged from $0.3 \%$ to $55 \%{ }^{12)}$ The site where intracranial arachnoid granulation occurs is diverse. Osma, et al., ${ }^{13)}$ reported that abnormal arachnoid granulation was originated from the posterior cranial fossa of the temporal bone at the range of $8.5 \%$. According to Yew, et al., ${ }^{14)}$ they reported that arachnoid granulation, which penetrated the dura and directly touched the matrix, had been detected at the range of $12.7 \%$. They described that with the range of more than $13 \%$, the arachnoid granulation incidence rate was higher in the posterior cranial fossa than the that in middle cranial fossa. They also stated that the brain tissue herniation occurs from large defect when arachnoid granulation is formed in the middle cranial fossa. The incidence rate of arachnoid granulation is higher in older age. $^{14)}$

A clinical suspicion is given a great weight on diagnosis of CSF otorrhea. If CSF otorrhea is suspected with the middle ear effusion, a defect of tegmen or arachnoid granulation can be confirmed through a radiologic examination. ${ }^{15)}$ According to Stone, et al., ${ }^{16)}$ non-enhanced high resolution CT (HRCT) was a way better diagnostic tool than enhanced CT cisternography or radionuclide cisternography. Moreover, they reported that the bony defect was detected through HRCT in 30 (71\%) of 42 patients with CSF leakage.

If a patient comes with watery otorrhea which is suspected to be CSF, $\beta-2$ transferrin assay would be beneficial for definite diagnosis of CSF otorrhea. $\beta$ - 2 transferrin analysis suggests high sensitivity and specificity for CSF. ${ }^{17)}$ Another useful test is a glucose test of the fluid being suspected to be CSF. When a patient, who is within the normal range of glucose level, has glucose level of more than $30 \mathrm{mg} / \mathrm{dL}$ of the suspected fluid, the fluid can be properly regarded as $\mathrm{CSF}^{15)}$ In our case, the fluid aspirated in the middle ear cavity indicated glucose level of $88 \mathrm{mg} / \mathrm{dL}$; thus, certainly we could consider it as CSF otorrhea.

Managements for CSF otorrhea principally target to prevent recurrent leakage of CSF. Moreover, when the disorder is accompanied with the brain tissue herniation, reduction of the herniated brain tissue and improvement of the patients' hearing sense are the ideal managements. In order to correct CSF leakage through the middle ear cavity, performing only middle cranial fossa approach method or using middle cranial fossa approach method with transmastoid approach is the most effective method. Since middle cranial fossa approach method provides a wide operation field of vision, it is profitable when there are many defects. ${ }^{15)}$ According to Gacek, et al., ${ }^{5)}$ the defect of middle cranial fossa was mostly managed through craniotomy with temporalis fascia. That of the posterior fossa was closed by approaching transmastoid method with the use of the adipose tissue. When performing transmastoid approach method, it has an advantage of using neither craniotomy nor temporal lobe retraction. However, it is difficult to approach the area when the defect is large or multiple or the defect is in the petrous apex. In our case, we tried to manage it by using transmastoid approach, because on the CT scan, the bony defect of the posterior wall of temporal bone was noted to be single.

When we assess a patient without specific past medical history who complains of recurrent ear fullness and otorrhea, although it is a rare disorder, we should pay attention to the presence of CSF otorrhea. When the bone defect is found in a radiologic examination, the CSF leakage should be managed through adequate approach methods, considering the characteristics of the defect, such as its location and number.

\section{REFERENCES}

1) Wetmore SJ, Herrmann P, Fisch U. Spontaneous cerebrospinal fluid otorrhea. Am J Otol 1987;8:96-102.

2) Canfield R. Some conditions associated with the loss of cerebrospinal fluid. Ann Otolaryngol 1913;22:604-22.

3) Nahas Z, Tatlipinar A, Limb CJ, Francis HW. Spontaneous meningoencephalocele of the temporal bone: clinical spectrum and presentation. Arch Otolaryngol Head Neck Surg 2008;134:509-18.

4) Gacek RR. Arachnoid granulation cerebrospinal fluid otorrhea. Ann Otol Rhinol Laryngol 1990;99:854-62.

5) Gacek RR, Gacek MR, Tart R. Adult spontaneous cerebrospinal fluid otorrhea: diagnosis and management. Am J Otol 1999;20:770-6.

6) Ferguson BJ, Wilkins RH, Hudson W, Farmer J Jr. Spontaneous CSF otorrhea from tegmen and posterior fossa defects. Laryngoscope 1986; 96:635-44.

7) Neely JG. Classification of spontaneous cerebrospinal fluid middle ear effusion: review of forty-nine cases. Otolaryngol Head Neck Surg 1985;93:625-34.

8) Kaufman B, Nulsen FE, Weiss MH, Brodkey JS, White RJ, Sykora GF. Acquired spontaneous, nontraumatic normal-pressure cerebrospi- 
nal fluid fistulas originating from the middle fossa. Radiology 1977; 122:379-87.

9) Potts DG, Reilly KF, Deonarine V. Morphology of the arachnoid villi and granulations. Radiology 1972;105:333-41.

10) Gomez DG, Potts G, Deonarine V, Reilly KF. Effects of pressure gradient changes on the morphology of arachnoid villi and granulations of the monkey. Lab Invest 1973;28:648-57.

11) Kaufman B, Yonas H, White RJ, Miller CF 2 nd. Acquired middle cranial fossa fistulas: normal pressure and nontraumatic in origin. Neurosurgery 1979;5:466-72.

12) Casey SO, Ozsvath R, Choi JS. Prevalence of arachnoid granulations as detected with CT venography of the dural sinuses. AJNR Am J Neuroradiol 1997;18:993-4.

13) Osma U, Cureoglu S, Hosoglu S. The complications of chronic otitis media: report of 93 cases. J Laryngol Otol 2000;114:97-100.
14) Yew M, Dubbs B, Tong O, Nager GT, Niparko JK, Tatlipinar A, et al. Arachnoid granulations of the temporal bone: a histologic study of dural and osseous penetration. Otol Neurotol 2011;32:602-9.

15) Brown NE, Grundfast KM, Jabre A, Megerian CA, O’Malley BW Jr, Rosenberg SI. Diagnosis and management of spontaneous cerebrospinal fluid-middle ear effusion and otorrhea. Laryngoscope 2004;114: $800-5$.

16) Stone JA, Castillo M, Neelon B, Mukherji SK. Evaluation of CSF leaks: high-resolution CT compared with contrast-enhanced CT and radionuclide cisternography. AJNR Am J Neuroradiol 1999;20: 706-12.

17) Skedros DG, Cass SP, Hirsch BE, Kelly RH. Beta-2 transferrin assay in clinical management of cerebral spinal fluid and perilymphatic fluid leaks. J Otolaryngol 1993;22:341-4. 